\title{
La disincronía cardíaca se correlaciona con el remodelado ventricular izquierdo postinfarto agudo al miocardio
}

\author{
Paul McN ab, Pablo Castro, Luigi G abrielli, Hugo Verdejo, \\ Juan Carlos Q uintana, José A Rodríguez, Ramón Corbalán \\ Cardiac dyssynchrony correlates \\ with left ventricular remodeling \\ after myocardial infarction
}

Background: Cardiac dyssynchrony is common in advanced heart failure (HF), but the changes in cardiac synchrony after myocardial infarction (MI) have not been adequately described. Aim: To study the relationship between cardiac synchrony and left ventricular remodeling after acute myocardial infarction. Material and methods: Forty nine patients aged $59 \pm 10$ years ( $77 \%$ men) with a first episode of a ST segment elevation MI, were studied. Scintigraphic left ventricular function and synchrony analyses were performed at baseline and after a six months follow-up. Determinations were compared with 33 healthy subjects. Results: At baseline, patients with MI had a decreased left ventricular ejection fraction (LVEF) and significant dyssynchrony, when compared with controls. LVEF was $36.4 \% \pm 10 \%$, left ventricular end-diastolic volume (LVEDV) $127 \pm 38 \mathrm{~mL}$, interventricular delay (IEV) $29 \pm 35$ miliseconds (ms), and intraventricular delay (IAV), 234 \pm 89 ms. After 6 months, LVEF significantly improved $(38 \% \pm 10 \%, p=0.042)$ without significant changes in LVEDV $(129 \pm 32$ $\mathrm{mL}, \mathrm{p}=0.97)$, IEV $(24 \pm 17, \mathrm{p}=0.96)$ or IAV $(231 \pm 97, \mathrm{p}=0.34)$. At baseline there were significant correlations between IAV and LVEF, and between IAV and LVEDV ( $r=0.48, p=0.001$ and $r=0.41$, $\mathrm{p}=0.004$, respectively). These correlations remained significant after 6 months. There was a positive correlation between IAV and LVEDV changes at six months $(r=0.403, p=0.04)$. Conclusions: The development of cardiac dyssynchrony correlates with adverse left ventricular remodeling after MI (Rev Méd Chile 2009; 137: 1457-62).

(Key w ords: Cardiac dyssynchrony; Myocardial infarction; Ventricular remodeling)

Recibido el 30 de diciembre, 2008. Aceptado el 29 de septiembre, 2009.

Departamento de Enfermedades Cardiovasculares, Pontificia Universidad Católica de Chile. Santiago de Chile.

Correspondencia a: Dr. Pablo F. Castro. Departamento Enfermedades Cardiovasculares, Pontificia Universidad de Católica de Chile. Marcoleta 367, Santiago, Chile. Fax: (562) 633-3171. E mail: pcastro@med.puc.cl 
L a asincronía en la contracción miocárdica ha sido reconocida como un hallazgo frecuente en pacientes con insuficiencia cardíaca avanzada (IC) ${ }^{1}$. La terapia de resincronización cardíaca (TRC) mejora la capacidad funcional, la función ventricular izquierda y el pronóstico en pacientes con IC con deterioro de capacidad funcional moderada a severa, QRS prolongado y disfunción sistólica del ventrículo izquierdo ${ }^{2}$. Adicionalmente la TRC produce regresión del remodelado ventricular izquierdo, mejora la función sistólica y disminuye la insuficiencia mitral ${ }^{2,3}$.

Posterior a un infarto agudo al miocardio (IAM) se produce una disociación en el tiempo de contracción de los distintos segmentos del ventrículo izquierdo contribuyendo al remodelado y disfunción ventricular izquierda 4 .

Los cambios que se producen en la sincronía cardíaca posterior a un IAM no han sido descritos. El objeto del presente trabajo es evaluar el remodelado y la sincronía postinfarto en un período de 6 meses mediante ventriculografía radioisotópica.

\section{MÉTodos}

Se incluyeron 49 pacientes de manera consecutiva y prospectiva que ingresaron por IAM a nuestra Unidad Coronaria. Los criterios de inclusión fueron: (1) primer episodio documentado de IAM con supradesnivel del segmento ST definido por electrocardiograma (ECG) con supradesnivel del segmento ST mayor de $2 \mathrm{~mm}$ en dos o más derivadas contiguas y curva enzimática característica, (2) admisión dentro de las primeras $72 \mathrm{~h}$ de iniciado los síntomas, (3) ventriculografía radioisotópica dentro de la primera semana del infarto y a los 6 meses de seguimiento. Se excluyeron pacientes con: (1) antecedente de IAM previo, (2) enfermedad valvular significativa conocida, (3) shock cardiogénico, (4) nivel de creatinina mayor de $2 \mathrm{mg} / \mathrm{dl}$, (5) cirugía de revascularización miocárdica dentro de la evolución intrahospitalaria, (6) presencia de marcapaso transitorio o definitivo, (7) bloqueo completo de rama derecha o izquierda en ECG de ingreso. Todos los pacientes firmaron un consentimiento informado aprobado por el Comité de Ética de nuestra institución.
Una cohorte de referencia de 33 sujetos sanos pareados por edad y sexo se utilizó como comparación de las medidas cintigráficas basales.

Durante la hospitalización todos los pacientes recibieron terapia considerada óptima para su condición por sus cardiólogos tratantes. Las terapias de reperfusión incluidas fueron angioplastia y trombólisis.

Se marcaron los glóbulos rojos autólogos con técnica in vitro con 740 a $925 \mathrm{MBq}$ de $\mathrm{Tc}^{99 \mathrm{~m}}$. Para la obtención de las imágenes se utilizó una gammacámara SPECT digital de doble detector (ADAC Forte, Milpitas, California) con un colimador de baja energía. Luego de 30 min de redistribución, se realizó una adquisición gatillada mediante ECG. El ciclo cardíaco fue dividido en 20 segmentos temporales que consideran en total $90 \%$ del intervalo $\mathrm{R}-\mathrm{R}$, con una tolerancia para rechazo de latidos de $20 \%$ en la longitud del mismo. Se obtienen 500 ciclos o $10 \mathrm{~min}$ de adquisición en proyección septal anterior con la mejor separación de cavidades cardíacas, típicamente en posición oblicua anterior izquierda.

Un observador experimentado definió las áreas de interés en forma automática con corrección manual de los bordes sobre el ventrículo izquierdo y derecho. Se calculó la fracción de eyección y volumen de fin de diástole del ventrículo izquierdo (FEVI y VFDVI) utilizando un software dedicado (Mirage v. 5.4 Segami Corporation, Columbia, MD). Las curvas de tiempo/volumen ventricular fueron derivadas utilizando transformación de Fourier de cuarto orden.

Se realizó análisis de fase sobre los datos cintigráficos consistente en el estudio de cada coordenada espacial de la imagen, en el tiempo del intervalo $\mathrm{RR}$, mediante transformación de Fourier. De la primera derivada de la transformación se calcula el ángulo de fase para cada coordenada, la que es codificada por color en una imagen paramétrica denominada simplemente "imagen de fase". Esta imagen representa la secuencia relativa y al patrón de contracción ventricular durante el ciclo cardíaco. La segunda imagen que se obtiene del análisis de fase se denomina "imagen de amplitud" proveniente del segundo parámetro del análisis armónico de Fourier y representa -como su nombre lo indicala amplitud de la curva tiempo/actividad en esa coordenada en particular y por lo tanto la magni- 
tud del "movimiento" en ese punto. Finalmente se calculó el retraso interventricular (RIT) definido como la diferencia absoluta del ángulo de fase modal de cada ventrículo y el retraso intraventricular (RIV) como la desviación estándar de la fase del ventrículo izquierdo.

Las variables continuas se expresan como promedios \pm DE y se comparan mediante el test de suma de rangos de Wilcoxon para muestras pareadas. Las variables categóricas se expresan como porcentajes. Los métodos de Pearson y Spearman fueron utilizados para evaluar correlaciones. Las determinaciones fueron comparadas con un grupo control. Se utilizó un modelo lineal general para evaluar interacciones y confirmar la independencia entre el cambio del VFDVI y el RIV a los 6 meses. Se consideró significativo un valor $\mathrm{p}<0,05$. Para todos los análisis se utilizó el programa estadístico SPSS 12.0 (SPSS Inc., Chicago, Illinois).

\section{Resultados}

Se incluyeron 49 pacientes consecutivos con IAM, $77 \%$ hombres, la edad promedio fue $59 \pm 9,6$ años, $14 \%$ eran diabéticos y $30,6 \%$ estaban en control por hipertensión arterial (Tabla 1). La mayoría de los pacientes se encontraban en Killip I $(73,5 \%)$ o
II $(24,5 \%)$ al ingreso. El infarto fue de pared anterior en la mayoría de los casos (77,6\%). Cerca de la mitad fueron elegibles para terapia de reperfusión, principalmente por criterios de temporalidad desde el inicio de los síntomas. Se realizó trombólisis en diecinueve pacientes y nueve fueron tratados con angioplastia primaria. Todos los pacientes recibieron el resto de la terapia médica recomendada para el manejo del IAM.

Los pacientes con IAM presentaban una FEVI disminuida y una disincronía significativa comparado con los controles, dado por un RIV y RTT significativamente mayores (Tabla 1). Basalmente, en los pacientes con IAM, la FEVI fue de 36,4 \pm $10 \%$, el VFDVI fue de $127 \pm 38 \mathrm{~mL}$, el RTT fue de 29 \pm 35 mseg (rango 0-197 ms) y el RIV fue de $234 \pm$ 89 mseg (rango 85-559 mseg). Después del seguimiento de 6 meses se produjo una mejoría significativa de la fracción de eyección de 36,4\% $\pm 10 \%$ a $38 \% \pm 10 \%(p=0,042)$, sin cambios significativos en el VFDVI $(127 \pm 38$ vs $129 \pm 32 \mathrm{~mL}, \mathrm{p}=0,97)$, RIT ( $29 \pm 35$ mseg vs $24 \pm 17$ mseg, $p=0,96)$ ni en el RIV ( $234 \pm 89$ vs $231 \pm 97$ mseg, $p=0,11$ ).

En las mediciones basales de los pacientes con IAM hubo una correlación significativa entre el RIV y la FEVI y entre el RIV y la VFDVI $(r=-0,48$; $\mathrm{p}=0,001$ y $\mathrm{r}=0,41 ; \mathrm{p}=0,004$, respectivamente). Además hubo una correlación significativa entre el

\section{Tabla 1. C aracterísticas basales de los pacientes con infarto agudo al miocardio y sujetos controles}

\begin{tabular}{|c|c|c|c|}
\hline & $\begin{array}{l}\text { Cohorte de estudio } \\
\qquad(n=49)\end{array}$ & $\begin{array}{l}\text { Controles } \\
(n=33)\end{array}$ & $\mathbf{p}$ \\
\hline Edad, años (promedio \pm DE) & $59,4 \pm 9,5$ & $58,8 \pm 10$ & NS \\
\hline Sexo masculino (\%) & 77,6 & 75,8 & NS \\
\hline Diabetes mellitus (\%) & 14,2 & N/A & N/A \\
\hline Hipertensión (\%) & 30,6 & $\mathrm{~N} / \mathrm{A}$ & N/A \\
\hline Killip I (\%) & 73,5 & $\mathrm{~N} / \mathrm{A}$ & N/A \\
\hline IAM pared anterior $(\%)$ & 77,6 & $\mathrm{~N} / \mathrm{A}$ & N/A \\
\hline Nivel máximo CK, UI/L (promedio $\pm \mathrm{DE}$ ) & $2917 \pm 1366$ & N/A & N/A \\
\hline Trombólisis (\%) & 38,8 & $\mathrm{~N} / \mathrm{A}$ & N/A \\
\hline Angioplastia primaria & 18,4 & N/A & N/A \\
\hline FEVI, \% (promedio $\pm \mathrm{DE})$ & $36,5 \pm 10,7$ & $55,8 \pm 4,7$ & $<0,01$ \\
\hline VFDVI, mL (promedio \pm DE) & $127,6 \pm 38,5$ & $100,5 \pm 23,2$ & $<0,01$ \\
\hline RIV, mseg (promedio $\pm \mathrm{DE}$ ) & $234 \pm 89$ & $78,2 \pm 24,0$ & $<0,01$ \\
\hline RIT, mseg (promedio $\pm \mathrm{DE}$ ) & $29 \pm 35$ & $24 \pm 17$ & $<0,01$ \\
\hline
\end{tabular}


RIT y la FEVI $(r=-0,35, p=0,01)$. A los 6 meses estas correlaciones se mantuvieron significativas (Tabla 2). Por otro lado el RIV se correlacionó positivamente con el grado de dilatación del VI ( $\mathrm{r}$ $=0,403 ; \mathrm{p}=0,04$ ) (Figura 1).

Los cambios de la FEVI en la evolución postinfarto presentaron una correlación significativa con los cambios en el RIV y en el VFDVI. Esta última correlación en los pacientes con infarto de pared anterior y en los pacientes con disfunción sistólica basal definido por una FEVI $<50 \%$ se mantiene significativa, ( $\mathrm{r}=0,409, \mathrm{p}=0,01)$ y ( $\mathrm{r}$ $=0,445, \mathrm{p}=0,04)$, respectivamente. Estas correlaciones se mantuvieron significativas luego del ajuste por sexo, edad, peak de creatinkinasa, terapia de reperfusión y clasificación de Killip en un modelo lineal general.

\section{DISCUSIÓN}

La IC es una condición que está en continuo aumento y para lo cual las herramientas terapéuticas actuales, solamente nos permiten un alivio parcial de esta patología ${ }^{3}$. La enfermedad coronaria es la principal causa de IC en el mundo desarrolla$\mathrm{do}^{4}$. Estudios recientes han mostrado que un deterioro en la coordinación mecánica de la contracción del ventrículo izquierdo es un hallazgo común en los pacientes con IC, particularmente en el contexto de un QRS ensanchado 5 ,6. De esta forma la disincronía cardíaca está relacionada a un aumento en el riesgo de falla ventricular, arritmias ventriculares malignas y a una mayor mortalidad 7,8 .

En el contexto de esta situación clínica, la evaluación de la sincronía cardíaca radica en el

Tabla 2. C orrelación entre parámetros cintigráficos de sincronía y remodelado ventricular izquierdo

\begin{tabular}{|lcccc|}
\hline & RIV basal & RIT basal & RIV 6 meses & RIT $\mathbf{6}$ meses \\
\hline FEVI & $-0,48^{*}$ & $-0,35^{*}$ & $-0,61^{*}$ & $-0,35^{*}$ \\
VFDVI & $0,41^{*}$ & $-0,12$ & $0,41^{*}$ & $-0,02$ \\
\hline
\end{tabular}

$*_{p}<0,05$, correlación de Pearson.

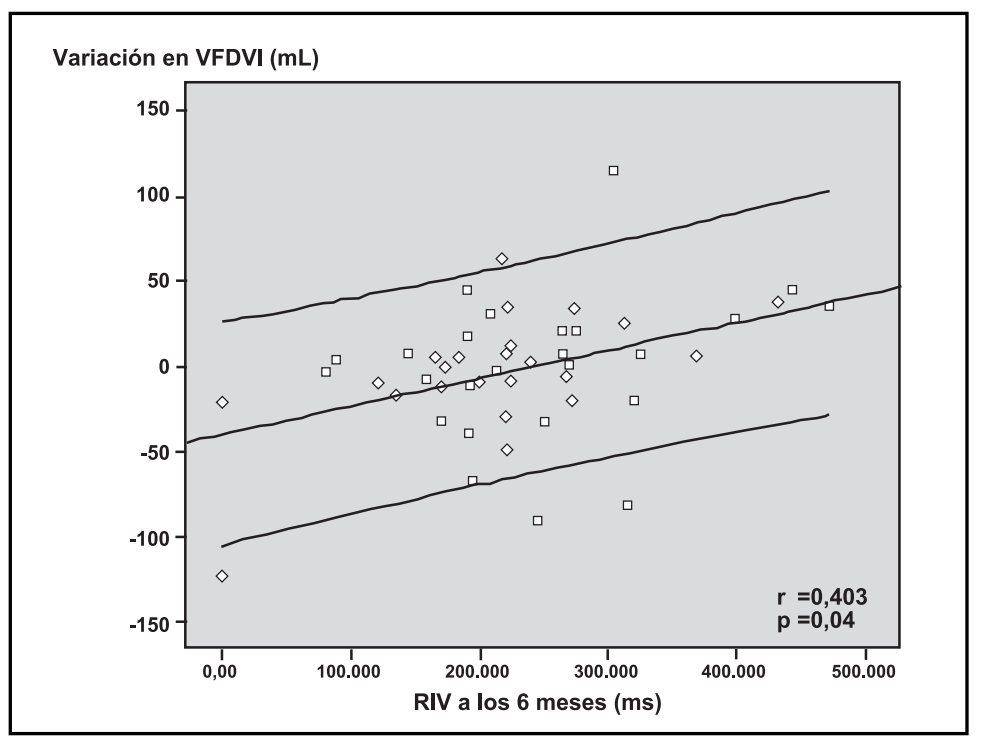

Figura 1. Correlación entre el cambio en el VFDVI y el retardo en la conducción intraventricular a los 6 meses. 
análisis de las paredes ventriculares basado en métodos de la ecocardiografía bidimensional, lo cual deriva fundamentalmente del análisis de movimientos longitudinales. La elección de la orientación está fundamentalmente basada en razones prácticas dadas por las ventanas disponibles para el posicionamiento del transductor. Sin embargo, el movimiento cardíaco es básicamente circunferencial. Recientemente, el análisis mediante ventriculografía con radionucleótidos se ha convertido en un método atractivo para evaluar la sincronía ventricular izquierda, ya que permite examinar todos los segmentos del ventrículo izquierdo de forma simultánea ${ }^{9,10}$.

Durante la oclusión aguda de una arteria coronaria, el patrón fisiológico del llenado y contracción ventricular se altera, apareciendo cambios característicos llamados hipokinesia/akinesia o diskinesia del área comprometida con hiperkinesia compensatoria de las regiones no afectadas ${ }^{11}$. Después de la resolución del período isquémico agudo, el miocardio afectado sufre un proceso conocido como remodelado cardíaco, incluyendo dilatación ventricular izquierda progresiva, entre otros cambios. En un reporte previo

\section{REFERENCIAS}

1. Rodríguez JA, Godoy I, Castro P, Quintana JC, Chávez E, Yovanovich J ET AL. Ramipril vs espironolactona en el remodelamiento ventricular izquierdo postinfarto: estudio randomizado y doble ciego. Rev Méd Chile 1997; 125: 643-52.

2. Corbalán R, Prieto JC, Chávez E, Nazzal C, Cumsile F, KRUCOFF M. Bedside markers of coronary artery patency and short-term prognosis of patients with acute myocardial infarction and thrombolysis. Am Heart J 1999; 138: 533-9.

3. YANCY CW. Comprehensive treatment of heart failure: state-of-the art medical therapy. Rev Cardiovasc Med 2005; 6 Suppl 2: S43-57.

4. Kiein L, Gheorghiade M. Coronary artery disease and prevention of heart failure. Med Clin North Am 2004; 88: 1209-35.

5. Fauchier L, Marie O, Casset-Senon D, Babuty D, COSNAY P, FAUCHIER JP. Reliability of QRS duration and morphology on surface electrocardiogram to identify ventricular dyssynchrony in patients with idiopa- de nuestro grupo se mostró una correlación positiva entre el VFDVI y la disincronía ventricular izquierda en pacientes con IC, independiente de la etiología de su condición. Por otro lado el uso de carvedilol en estos pacientes resultó en una mejoría en la sincronía cardíaca, lo que en parte puede estar explicado por una reducción en el VFDVI $^{12}$. El presente estudio confirma la correlación entre el remodelado ventricular post IAM y la disincronía ventricular izquierda basalmente y a los 6 meses de seguimiento. Esta correlación se mantiene luego de ajustar por: edad, sexo, tamaño del infarto y terapia de reperfusión. La relación entre disincronía y remodelado ventricular izquierdo está establecido en pacientes con IC ${ }^{13-17}$, nuestros hallazgos apoyan esta relación en pacientes post IAM.

En conclusión, nuestros resultados muestran una correlación entre el remodelado ventricular y el desarrollo de disincronía ventricular post IAM mediante la utilización de ventriculografía con radionucleótidos. Esta técnica podría constituir una alternativa al estudio ecocardiográfico y contribuir a identificar los pacientes susceptibles de intervención.

thic dilated cardiomyopathy. Am J Cardio 2003; 92: 341-4.

6. Ghio S, Constantin C, Klersy C, Serio A, Fontana A, CAMPANA C ET AL. Interventricular and intraventricular dyssynchrony are common in heart failure patients, regardless of QRS duration. Eur Heart J 2004; 25: 571-8.

7. BaLdasseroni S, Opasich C, Gorini M, Lucci D, MarchioNNI N, Marini M ET aL. Italian Network on Congestive Heart Failure Investigators. Left bundle branch block is associated with increased 1 year sudden and total mortality rate in 5517 outpatients with congestive heart failure: a report from the Italian network on congestive heart failure. Am Heart J 2002; 143: 398405.

8. Luliano S, Fischer SG, Karasik PE, Fletcher RD, Singh SN; Departament of Veterans Affairs Survival Trial of Antiarrhythmic Therapy in Congestive Heart Failure. QRS duration and mortality in patients with congestive heart failure. Am Heart J 2002; 143: 1085-91.

9. Fauchier L, Marie O, Casset-Sennon D, Babuty D, COSNAY P, FAUCHIER JP. Interventricular and intraventri- 
cular dyssynchrony in idiopathic dilated cardiomyopathy: a prognostic study with Fourier phase analysis of radionuclide angioscintigraphy. J Am Coll Cardiol 2002; 40: 2022-30.

10. Kerwin WF, Botvinick EH, O'Conell JW, MerRICK SH, DeMarco T, Chatterjee K et AL. Ventricular contraction abnormalities in dilated cardiomyopathy: effect of biventricular pacing to correct interventricular dyssynchrony. J Am Coll Cardiol 2000; 35: 1221-7.

11. Bruch C, Bartel T, Schmermund A, Schatr J, Erbel R. Asynchronie der ventrikularen Kontraktion und Relaxation- patophysiologisch erkanntes Phänomen, jetzt klinisch nachweisbar. Hertz 1998; 23: 506-15.

12. Castro PF, McNab P, Quintana JC, Bittner A, Greig D, VERGARA I ET AL. Effects of carvedilol upon intra and interventricular synchrony in patients with chronic heart failure. Am J Cardiol 2005; 96: 267-9.

13. Duncan A, Francis D, Gibson D, Pepper J, Henein M. Electromechanical left ventricular resynchronization by coronary artery by-pass surgery. Eur J Cardio Thoracic Surg 2004; 26: 711-9.

14. Leclero C, Gras D, Tang A, Alonso C, Thomas-Revault D’Alones F, Mabo P. InSync Study Group. Comparati- ve effects of ventricular resynchronization therapy in heart failure patients with or without coronary artery disease. Ann Cardio Angeio 2004; 53: 171-6.

15. Barbieri, Bursi F, Bonatti S, Coppi F, Zanasi V, REgGianini L ET AL. Evidence of reverse remodeling after long-term biventricular stimulation for resynchronization in patients with wide QRS selected on the basis of echocardiographic electromechanical delays. Ita Heart J 2004; 5: 818-25.

16. Bristow MR, Saxon LA, Boehmer J, Krueger S, Kass DA, De Marco T ET aL. Comparison of Medical Therapy, Pacing and Defibrillation in Heart Failure (COMPANION) Investigators. Cardiac-resynchronization therapy with or without an implantable defibrillator in advanced chronic heart failure. N Eng J Med 2004; 350: 2140-50.

17. Clemand JG, Daubert JC, Erdmann E, Freemantle N, Gras D, KaPPEnBERger L ET AL. Cardiac Resynchronization-Heart Failure (CARE-HF) Study Investigators .The effect of cardiac resynchronization in morbidity and mortality of heart failure. N Eng J Med 2005; 352: 1539-49.

\section{ABREVIACIONES}

FEVI: $\quad$ Fracción eyección ventrículo izquierdo

VFDVI: Volumen de fin de diástole ventrículo izquierdo

RIV: $\quad$ Retardo conducción intraventricular

RIT: $\quad$ Retardo conducción interventricular

mseg: $\quad$ Milisegundos 